\title{
Evaluation of the effect of flavangenol on serum lipid peroxide levels and development of atherosclerosis in spontaneously hyperlipidemic B6.KOR-Apoe $e^{\text {shl }}$ mice
}

\author{
KOUICHI SUGAYA ${ }^{1}$, MAMORU IGARASHI $^{1}$, YUKO KOJIMA $^{2}$, MASAHITO TSUBATA $^{3}$ and ISAO NAGAOKA ${ }^{1}$ \\ ${ }^{1}$ Department of Host Defense and Biochemical Research, ${ }^{2}$ Division of Biomedical Imaging Research, Biomedical Research \\ Center, Juntendo University, Graduate School of Medicine, Tokyo; ${ }^{3}$ Toyo Shinyaku Co., Ltd., Saga, Japan
}

Received August 12,2010; Accepted October 8, 2010

DOI: $10.3892 /$ ijmm.2010.554

\begin{abstract}
Antioxidative flavonoids are used to reduce the risk of cardiovascular diseases in humans. However, the precise mechanism for the anti-atherosclerotic actions of flavonoids remains to be elucidated. In the present study, to assess the mechanism for the action of antioxidative flavonoids on atherosclerosis, we investigated the effect of flavangenol, one of the most potent antioxidants currently known, on spontaneously hyperlipidemic B6.KOR-Apoeshl mice. Flavangenol was orally administered to B6.KORApoe $^{\text {shl }}$ mice ad libitum (6 mg flavangenol/mouse/day). After 6 months, serum levels of lipids (total cholesterol, triglyceride, HDL-cholesterol and LDL-cholesterol) and lipid peroxide were measured, and histopathological changes (lipid accumulation and inflammatory cell infiltration) in the aortic root were evaluated. Serum levels of total cholesterol and LDL-cholesterol were markedly increased, and HDLcholesterol levels were decreased in B6.KOR-Apoe ${ }^{\text {shl }}$ mice compared to $\mathrm{C} 57 \mathrm{BL} / 6$ mice used as a control $(\mathrm{p}<0.001)$. Among these serum lipids, only HDL-cholesterol levels were significantly increased by flavangenol administration $(\mathrm{p}<0.05)$. Moreover, Oil Red O staining (lipid accumulation) was significantly increased in B6.KOR-Apoe shl mice compared to C57BL/6 mice $(\mathrm{p}<0.001)$. Notably, flavangenol administration significantly suppressed the increase in Oil Red O staining $(\mathrm{p}<0.01)$. Similarly, inflammatory cell infiltration into the intima was significantly increased in B6.KOR-Apoeshl mice compared to C57BL/6 mice ( $\mathrm{p}<0.01)$, and flavangenol administration significantly suppressed the inflammatory cell infiltration $(\mathrm{p}<0.01)$. Importantly,
\end{abstract}

Correspondence to: Dr Isao Nagaoka, Department of Host Defense and Biochemical Research, Juntendo University, Graduate School of Medicine, 2-1-1 Hongo, Bunkyo-ku, Tokyo 113-8421, Japan E-mail: nagaokai@med.juntendo.ac.jp

Key words: pine bark extract, atherosclerosis, antioxidant, lipid peroxide, hyperlipidemic mice flavangenol administration significantly reduced the increase of serum lipid peroxide levels in B6.KOR-Apoeshl mice $(p<0.05)$. Together, these observations indicate that flavangenol, one of the most potent antioxidants, exerts its anti-atherosclerotic action on spontaneously hyperlipidemic and atherosclerotic B6.KOR-Apoeshl mice, possibly by increasing HDL-cholesterol levels and reducing lipid peroxide levels, thereby suppressing the lipid accumulation (formation of atherosclerotic lesions) and inflammatory cell infiltration (chronic inflammation) in the intima of the aortic root.

\section{Introduction}

Hypercholesterolemia, diabetes and tobacco are risk factors for the pathogenesis of atherosclerosis (1). In addition to these established risk factors, epidemiological studies have indicated that increased oxidative stress is also involved in atherosclerosis (2-4). Atherosclerosis lesions develop from a focal accumulation of lipoproteins and monocyte-derived macrophages within the wall of arterial blood vessels (5). Within the lesions, intimal macrophages promote the accumulation of cholesterol. The source of this cholesterol is lipoprotein particles that pass from the blood into the vessel wall and are subsequently modified by oxidation (5).

Flavangenol ${ }^{\circledR}$, also known as Pycnogenol ${ }^{\circledR}$, is a pine bark extract of the French maritime pine (Pinus pinaster) with a mixture of flavonoids, which are mainly compounds of proanthocyanidin containing the polyhydroxy flavan-3-ol unit in di- and tetra-form (6). Flavangenol is one of the most potent antioxidants currently known and is much more potent than the antioxidative vitamins $\mathrm{C}$ and $\mathrm{E}(6,7)$. In addition, flavangenol has antiplatelet and endothelial protective activities $(8,9)$. Of importance, flavangenol administration suppresses the development of atherosclerotic lesions in apolipoprotein E (ApoE)-knockout (KO) mice (10). Similarly, tea catechin and potato anthocyanins with antioxidative activities have been reported to suppress the formation of atherosclerotic lesions in ApoE-KO mice $(11,12)$. Thus, antioxidative flavonoids are used to reduce the risk of cardiovascular diseases in humans (13-15). However, in ApoE-deficient mice the levels of serum lipids (such as total cholesterol, LDL-cholesterol and HDL-cholesterol) and lipid peroxide are almost unchanged by the administration of 
antioxidative flavonoids; thus, the precise mechanism for the anti-atherosclerotic actions of flavonoids remains to be elucidated.

Spontaneously hyperlipidemic (SHL) mice (KOR-Apoe $e^{\text {shl }}$ ) are an inbred species established from Japanese wild-type mice (Mus musculus molossinus KOR) (16). Genetic analysis revealed gross disruption of the Apoe gene (a gene for ApoE) in KOR-Apoeshl, and SHL mice exhibit severe hypercholesterolemia (>1000 mg/dl) and develop atherosclerotic lesions, along with severe skin xanthoma. When compared with ApoE-KO mice, in which the Apoe gene has been disrupted by homologous recombination, cholesterol levels were much higher, but yet atherosclerotic lesions were less severe in SHL mice (17). Furthermore, three lines of congenic mice were established with a genetic background of C57BL/6, $\mathrm{BALB} / \mathrm{c}$ or $\mathrm{C} 3 / \mathrm{He}$ (17). Among SHL and congenic SHL mice, B6.KOR-Apoeshl mice exhibit the least severe hypercholesterolemia but develop the most severe atherosclerotic lesions (17).

In the present study, to elucidate the mechanism for the antioxidative actions of flavonoids on atherosclerosis, we investigated the effect of flavangenol, one of the most potent antioxidants currently known, on B6.KOR-Apoe ${ }^{\text {shl }}$ mice by evaluating serum levels of lipids (such as total cholesterol, HDL-cholesterol, LDL-cholesterol and triglyceride) and lipid peroxide as well as histopathological changes (lipid accumulation and inflammatory cell infiltration) in the aortic root.

\section{Materials and methods}

Animal model. All procedures were carried out according to the Institutional Animal Care and Committee Guide of the Juntendo University School of Medicine. Male B6.KORApoe ${ }^{\text {shl }}$ mice and C57BL/6 mice (8-week-old; Japan SLC, Shizuoka, Japan) were used in the present study. Three mice per cage were housed under specific pathogen-free conditions (controlled temperature of $24 \pm 3^{\circ} \mathrm{C}$ and humidity of $55 \pm 15 \%$ ) and fed standard laboratory food ad libitum.

Flavangenol ${ }^{\circledR}$ was supplied by Toyo Shinyaku Co., Ltd. (Saga, Japan). Flavangenol was dissolved in tap water $(1 \mathrm{mg} / \mathrm{ml})$ and orally administered to B6.KOR-Apoe ${ }^{\text {shl }}$ mice ad libitum for 6 months. The intake volume of flavangenol solution was measured once a week, and it was found that the animals received an average of $6 \mathrm{ml}$ flavangenol solution $(6 \mathrm{mg}$ flavangenol/mouse/day).

Mice were divided into three groups; the $\mathrm{C} 57 \mathrm{BL} / 6$ control group (C57BL/6; n=3), the B6.KOR-Apoe ${ }^{\text {shl }}$ group without flavangenol (B6.KOR-Apoeshl; $\mathrm{n}=3$ ) and the B6.KOR-Apoe $e^{\text {shl }}$ group with flavangenol (+Flavangenol; $n=3)$. All groups of mice were allowed to move freely in aluminum cages. Animals were sacrificed after 6 months.

Tissue preparation and histopathological evaluation. Mice were sacrificed by collecting blood from the inferior vena cava. Vasculature was perfused with phosphate-buffered saline (PBS; $30 \mathrm{ml}$ ) and 10\% formalin in PBS (30 ml). The aortic root was dissected under a microscope and frozen in optimal cutting temperature (OCT) embedding medium (Sakura Finetechnical, Co., Ltd., Tokyo, Japan). OCT- embedded tissue samples were sectioned at $3 \mu \mathrm{m}$. To analyze the atherosclerotic lesion in the aortic root, the sections were immersed for $15 \mathrm{sec}$ in $60 \%$ isopropanol, stained for $30 \mathrm{~min}$ in saturated Oil Red $\mathrm{O}$ solution at $37^{\circ} \mathrm{C}$, and then coverslipped with glycerol gelatin. Oil Red O-stained areas (atherosclerotic lesions) in the intima were measured as digital images by the K400 image analysis system (Carl Zeiss, Oberkohen, Germany). Furthermore, to analyze the inflammatory cell infiltration into the atherosclerotic lesions, sections were stained with hematoxylin and eosin. Three visual fields (x200) were randomly selected in each section, and infiltrated cells were counted under a microscope (Olympus, Japan) and averaged.

Measurement of serum lipids. Collected blood was centrifuged, and sera were stored at $-80^{\circ} \mathrm{C}$ until use. Serum total cholesterol, HDL-cholesterol and triglyceride levels were measured using commercially available enzyme assay kits (Wako Pure Chemicals, Tokyo, Japan) according to the manufacturer's instructions. LDL-cholesterol levels were calculated as [total cholesterol - (HDL-cholesterol + triglyceride/5)].

Measurement of serum lipid peroxide. Serum lipid peroxide levels were measured using a TBARS (thiobarbituric acid reactive substance) assay kit (Cayman Chemical, MI, USA) according to the manufacturer's instructions.

Statistical analyses. Data are presented as mean \pm SD. Statistical significance was determined by one way ANOVA or the Student's t-test (Prism 4, GraphPad Software, San Diego, CA). A p $<0.05$ was considered statistically significant.

\section{Results}

Evaluation of serum lipids. First, we evaluated the effect of flavangenol administration on serum lipid levels. Serum total cholesterol levels were $62.8 \pm 17.3 \mathrm{mg} / \mathrm{dl}$ in C57BL/6 mice, and markedly increased in B6.KOR-Apoeshl mice (625.8 \pm $94.8 \mathrm{mg} / \mathrm{dl})(\mathrm{p}<0.001)$; flavangenol administration did not affect total cholesterol levels $(617.2 \pm 88.8 \mathrm{mg} / \mathrm{dl})$ (Fig. 1A). Similarly, serum LDL-cholesterol levels were significantly increased in B6.KOR-Apoe ${ }^{\text {shl }}$ mice $(586.6 \pm 71.8 \mathrm{mg} / \mathrm{dl})$ compared to C57BL/6 mice $(2.9 \pm 1.2 \mathrm{mg} / \mathrm{dl})(\mathrm{p}<0.001)$; however, flavangenol administration did not have an effect on LDL-cholesterol levels (569.2 $\pm 73.3 \mathrm{mg} / \mathrm{dl})$ (Fig. 1B). In contrast, there were no significant differences in serum triglyceride levels among C57BL/6 mice $(98.8 \pm 8.3 \mathrm{mg} / \mathrm{dl})$, B6.KOR-Apoe ${ }^{\text {shl }}$ mice $(193.9 \pm 64.1 \mathrm{mg} / \mathrm{dl})$ and flavangenoladministered mice $(157.4 \pm 50.7 \mathrm{mg} / \mathrm{dl})$ (Fig. 1C). Of note, serum HDL-cholesterol levels were significantly decreased in B6.KOR-Apoe shl mice $(16.9 \pm 2.3 \mathrm{mg} / \mathrm{dl})$ compared to C57BL/6 mice $(53.7 \pm 6.9 \mathrm{mg} / \mathrm{dl})(\mathrm{p}<0.001)$, but were slightly increase by flavangenol administration (+Flavangenol; $22.0 \pm 1.7 \mathrm{mg} / \mathrm{dl}, \mathrm{p}<0.05$ ) (Fig. 1D).

Thus, total cholesterol and LDL-cholesterol levels markedly increased and HDL-cholesterol levels decreased in B6.KOR-Apoe ${ }^{\text {shl }}$ compared to C57BL/6 control mice. Among these serum lipids, only HDL-cholesterol levels were significantly increased by flavangenol administration. 
A
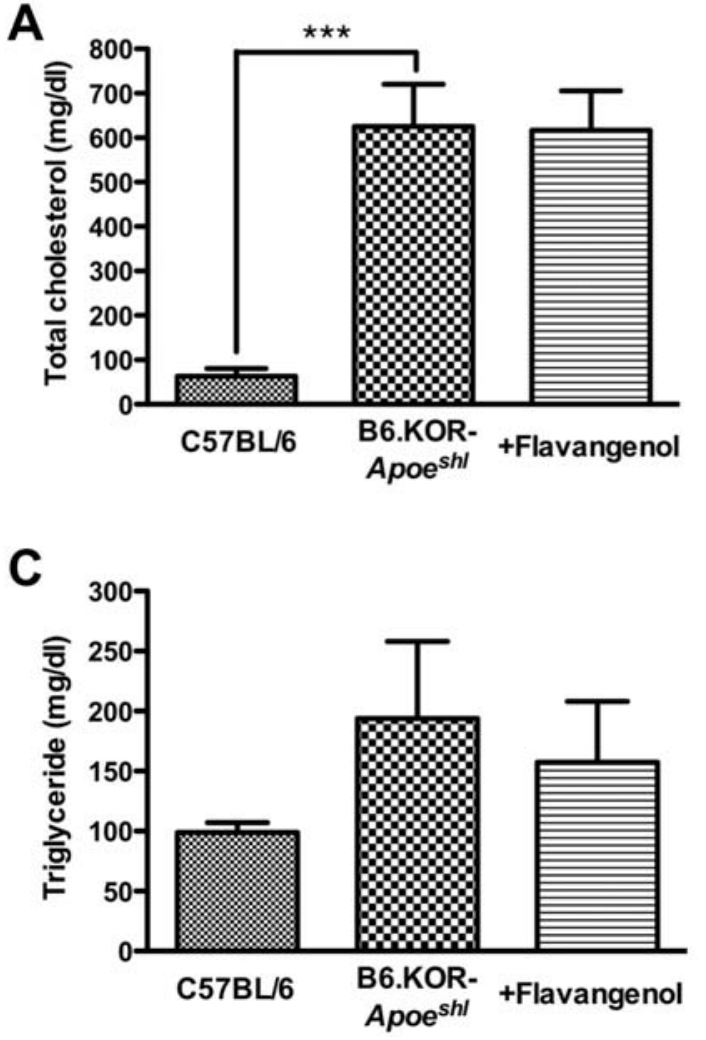

B

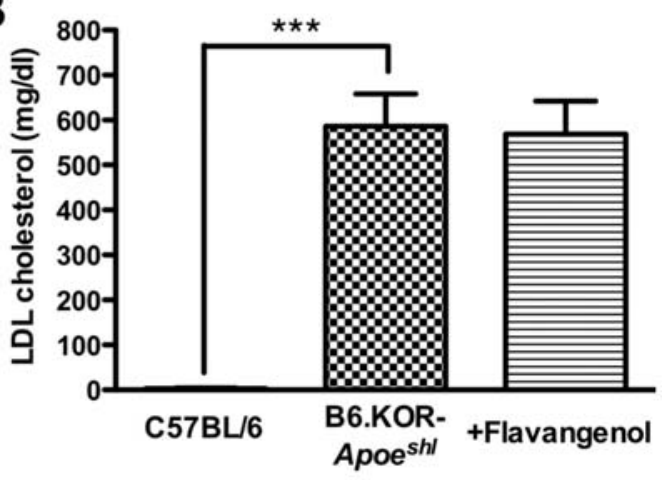

D

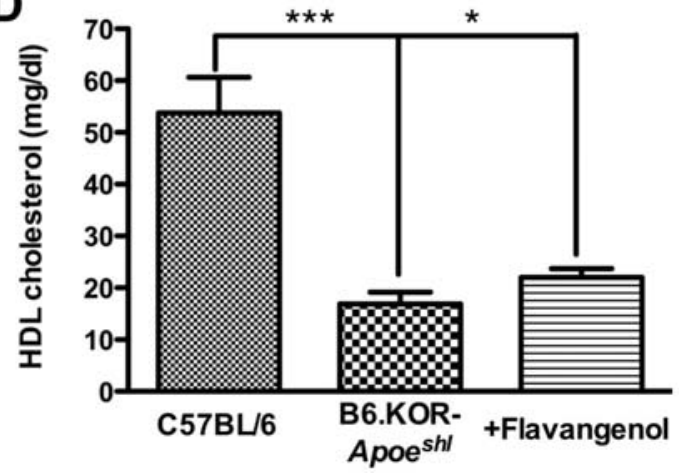

Figure 1. Effect of flavangenol administration on serum lipid levels. Serum samples were assayed for total cholesterol (A), LDL-cholesterol (B), triglyceride (C) and HDL-cholesterol (D). Data represent the mean \pm SD of three animals in C57BL/6 group, B6.KOR-Apoe ${ }^{\text {shl }}$ group and B6.KOR-Apoe ${ }^{\text {shl }}$ group with flavangenol administration (+Flavangenol). Values were compared between B6.KOR-Apoe ${ }^{\text {shl }}$ mice and C57BL/6 mice or + Flavangenol. ${ }^{*} \mathrm{p}<0.05,{ }^{* * *} \mathrm{p}<0.001$.

Effect of flavangenol administration on histopathological changes in atherosclerotic lesions. Next, we evaluated the effect of flavangenol administration on the histopathological changes of the atherosclerotic lesion in the aortic root by staining sections with Oil Red $\mathrm{O}$ and counting infiltrated cells in the atherosclerotic lesion. It has been reported that B6.KOR-Apoe ${ }^{s h l}$ mice spontaneously develop atherosclerotic lesions in the aortic root (13). Consistent with this, Oil Red O staining (i.e., lipid accumulation) was significantly increased in B6.KOR-Apoe ${ }^{\text {shl }}$ mice compared to C57BL/6 mice (Fig. 2A, $B$ and D) $(\mathrm{p}<0.001)$. Flavangenol administration significantly suppressed the increase of Oil Red O staining (Fig. 2C and D) $(\mathrm{p}<0.01)$. Similarly, inflammatory cell infiltration into the intimal and subintimal layers was significantly increased in B6.KOR-Apoe ${ }^{s h l}$ mice compared to C57BL/6 mice (Fig. 3A, $B$ and D) $(\mathrm{p}<0.01)$. Importantly, flavangenol administration significantly suppressed the increase in inflammatory cell infiltration (Fig. 3C and D) $(\mathrm{p}<0.01)$.

Thus, flavangenol exhibits an anti-atherosclerotic action in B6.KOR-Apoe ${ }^{\text {shl }}$ mice by suppressing lipid accumulation and inflammatory cell infiltration.

Effect of flavangenol administration on serum lipid peroxide level. Oxidative stress is associated with the pathogenesis of atherosclerosis (18). Thus, we evaluated the effect of flavangenol administration on serum lipid peroxide levels by the TBARS assay. The serum lipid peroxide levels were $9.2 \pm 0.6 \mathrm{nmol} / \mathrm{ml}$ in $\mathrm{C} 57 \mathrm{BL} / 6$ mice, and markedly increased in B6.KOR-Apoe shl mice $(41.2 \pm 3.1 \mathrm{nmol} / \mathrm{ml})$. Of note, flavangenol administration significantly reduced serum lipid peroxide levels in B6.KOR-Apoeshl mice $(32.6 \pm 3.4 \mathrm{nmol} / \mathrm{ml})$ (Fig. 4). These observations indicate that flavangenol administration suppresses the lipid peroxidation in B6.KORApoe $^{\text {shl }}$ mice.

\section{Discussion}

In the early stages of cardiovascular disease progression, endothelial dysfunction triggers a chronic inflammatory process in the vessel wall. Induction of adhesion molecules in endothelial cells facilitates the adhesion of monocytic leukocytes, trapping them and allowing their transmigration through the endothelial layer into the underlying intima. There they become tissue macrophages and subsequently foam cells following the uptake of oxidatively-modified lipids $(19,20)$. It is generally accepted that antioxidative flavonoids reduce the risk of cardiovascular diseases in humans $(12,13)$. However, the precise mechanism for the anti-atherosclerotic actions of flavonoids remains to be elucidated. Flavangenol is known to be one of the most potent antioxidants. In addition, flavangenol has antiplatelet and endothelial protective activities $(8,9)$. In this study, to elucidate the mechanism of the antioxidative effects of flavonoids on atherosclerosis, we investigated the effect of flavangenol, on SHL and atherosclerotic B6.KOR-Apoe ${ }^{\text {shl }}$ mice by evaluating serum levels of lipids and lipid peroxide as well as histopathological changes in the aortic root. 
A

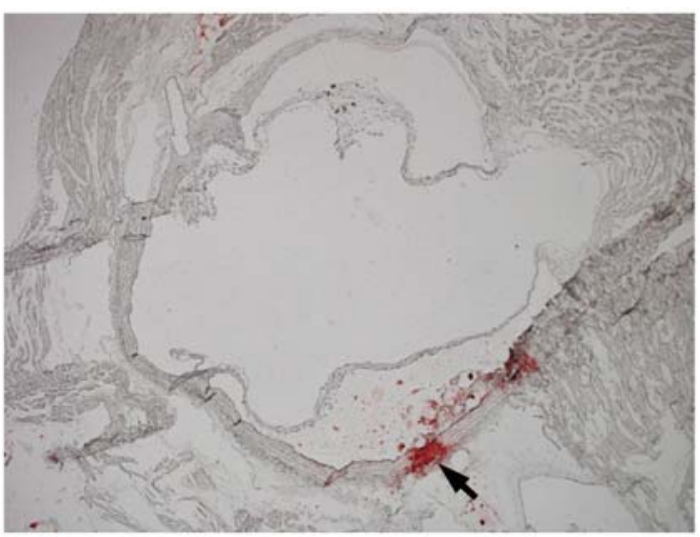

B

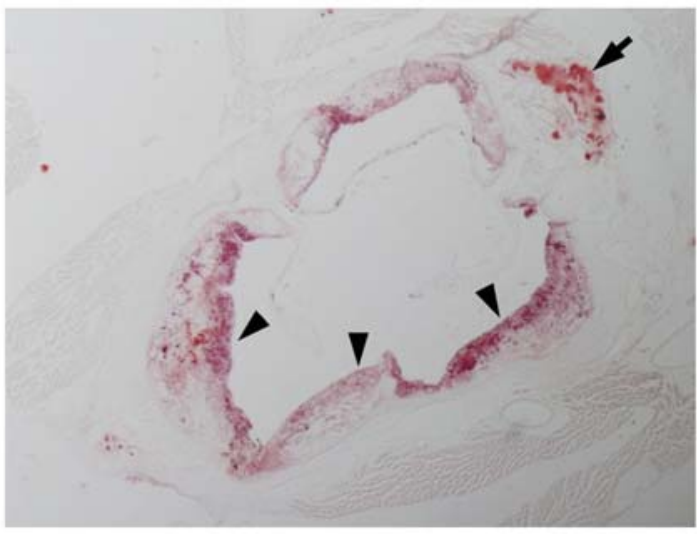

C

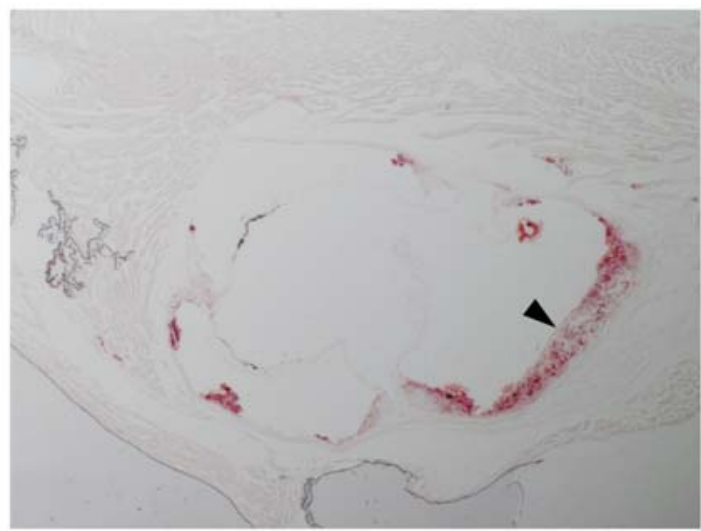

D

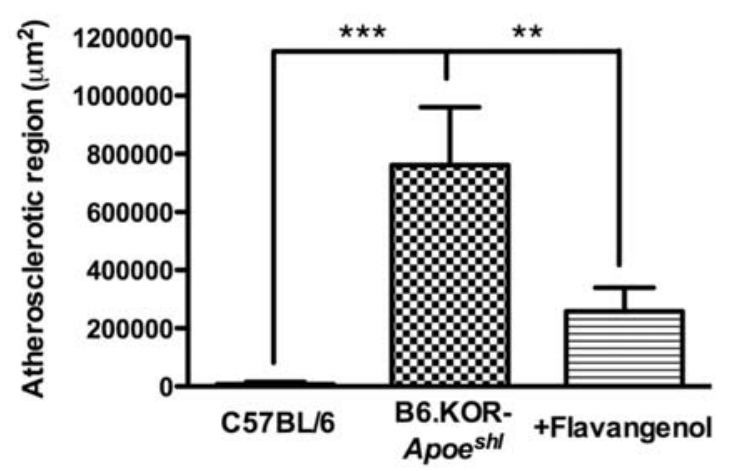

Figure 2. Effect of flavangenol administration on the lipid accumulation in the aortic root. Sections of (A) the aortic root from C57BL/6 group, (B) the B6.KOR-Apoe $e^{\text {shl }}$ group, and (C) the B6.KOR-Apoe $e^{\text {shl }}$ group with flavangenol administration were stained with Oil Red O (original magnification x40). Oil Red O-stained areas (atherosclerotic lesions) in the intima indicated by arrowheads were measured as digital images by the K400 image analysis system Arrows in panels A and B indicate nonspecific staining of the adventitia. (D) Data represent the mean \pm SD of three animals in the C57BL/6, B6.KOR-Apoeshl and B6.KOR-Apoe $e^{\text {shl }}$ with flavangenol administration (+Flavangenol) groups. Values were compared between B6.KOR-Apoe shl $^{\text {shl }}$ and C57BL/6 or +Flavangenol mice. ${ }^{* *} \mathrm{p}<0.01,{ }^{* * *} \mathrm{p}<0.001$.
A

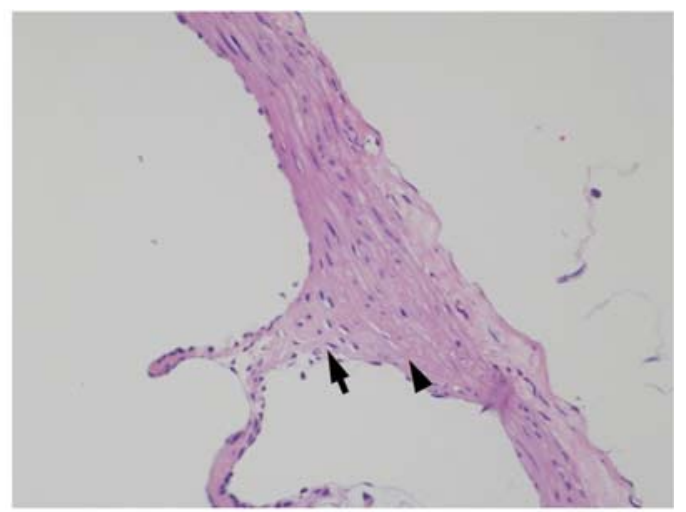

B

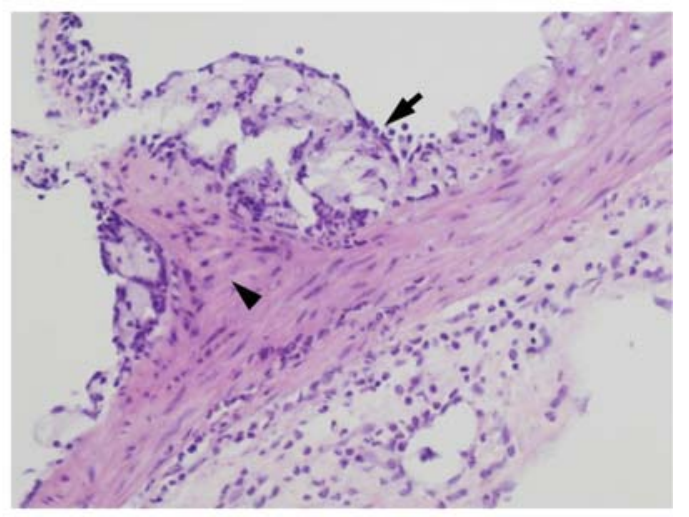

C

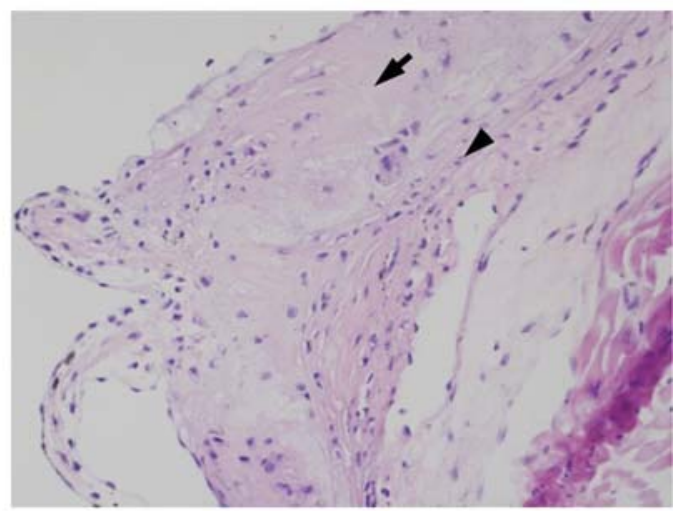

D

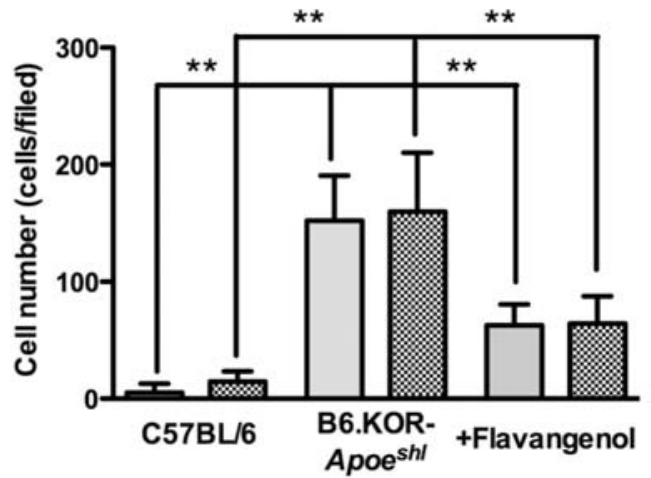

Figure 3. Effect of flavangenol administration on the inflammatory cell infiltration in the aortic root. Sections of the aortic root from (A) the C57BL/6, (B) B6.KOR-Apoe $e^{\text {shl }}$ and (C) B6.KOR-Apoe ${ }^{\text {shl }}$ with flavangenol administration groups were stained with hematoxylin and eosin (original magnification $\mathrm{x} 200$ ). Three visual fields were randomly selected in each section, and infiltrated inflammatory cells in the intimal and subintimal layers (indicated by arrows and arrowheads, respectively) were counted under a microscope and averaged (D). Data represent the mean \pm SD of three animals in the C57BL/6, B6.KOR-Apoe $e^{\text {shl }}$ and B6.KOR-Apoe ${ }^{\text {shl }}$ with flavangenol administration (+Flavangenol) groups. Values were compared between B6.KOR-Apoe ${ }^{s h l}$ and C57BL/6 or +Flavangenol mice in the intimal (hatched bars) and subintimal (stippled bars) layers. ${ }^{* *} \mathrm{p}<0.01$. 


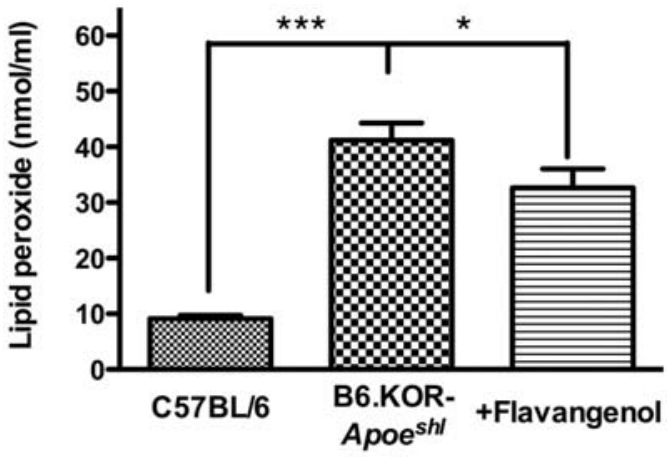

Figure 4. Effect of flavangenol administration on serum lipid peroxide levels. Serum samples were assayed for lipid peroxide using a TBARS assay kit. Data represent the mean \pm SD of three animals in the C57BL/6, B6.KORApoe $^{\text {shl }}$ and B6.KOR-Apoe ${ }^{\text {shl }}$ with flavangenol administration (+Flavangenol) groups. Values were compared between B6.KOR-Apoe $e^{\text {shl }}$ and C57BL/6 or +Flavangenol mice. ${ }^{*} \mathrm{p}<0.05,{ }^{* * *} \mathrm{p}<0.001$.

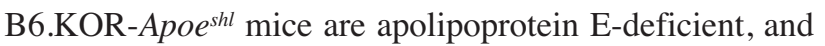
their dyslipidemic phenotypes, including their high plasma lipoprotein cholesterol levels and atherosclerosis, have been described by Matushima et al $(16,17)$. The present study confirmed that B6.KOR-Apoe ${ }^{\text {shl }}$ mice exhibit much higher levels of serum total cholesterol, triglyceride, LDL-cholesterol and lipid peroxide but lower levels of HDL-cholesterol than C57BL/6 mice used as a control. In addition, B6.KORApoeshl mice show a significant increase of Oil Red O staining and exhibit augmented inflammatory cell infiltration into the intimal and subintimal layers of the aortic root, compared to $\mathrm{C} 57 \mathrm{BL} / 6$ mice.

Dyslipidemia (hyperlipidemia) is one of the most important modifiable risk factors for cardiovascular diseases $(21,22)$. Thus, we examined the effect of flavangenol administration on the dyslipidemia observed in B6.KORApoe $^{\text {shl }}$ mice. Flavangenol administration did not have a significant effect on the increased levels of serum total cholesterol, triglyceride and LDL-cholesterol. In contrast, flavangenol administration slightly but significantly increased the reduced level of HDL-cholesterol. It has already been shown that HDL-cholesterol levels are inversely correlated with the risk of cardiovascular diseases, because increases in HDL-cholesterol decrease the risk of developing atherosclerosis (23). Thus, flavangenol is likely to suppress the development of atherosclerosis in B6.KOR-Apoe ${ }^{\text {shl }}$ mice by increasing HDL-cholesterol levels.

Oxidative stress is a risk factor for the development of cardiovascular disease, and it is involved in the pathogenesis and development of atherosclerosis (18). Lipid peroxidation, such as production of oxidative LDL-cholesterol, may occur under the conditions of hyperlipidemia, and oxidized substances, such as oxidized LDL-cholesterol, trigger inflammatory actions on endothelial cells and macrophages, and accelerate the progression of atherosclerosis (24). Of note, flavangenol administration significantly reduced the level of lipid peroxidation measured as TBARS, which is routinely assayed for the oxidative modification of LDL. Thus, flavangenol can reduce the oxidative stress (lipid peroxidation) and exhibit a suppressive action on the formation of atherosclerotic lesions in B6.KOR-Apoe ${ }^{\text {shl }}$ mice.
Atherosclerosis is considered to be a chronic inflammatory disease, which is characterized by lipid accumulation and infiltration of mononuclear leukocytes into the intima (23). In the present study, flavangenol administration not only reduced the area of Oil Red O-stained atherosclerotic lesions but also suppressed the infiltration of monocytic cells into the intima (chronic inflammation) in the aortic root of B6.KORApoe $^{\text {shl }}$ mice. These observations indicate that flavangenol represses the formation of atherosclerotic lesions (lipid accumulation) as well as the chronic inflammation of the vessel wall in dyslipidemia.

As for the effects on the atherosclerotic animal models, flavangenol administration (4.5 $\mathrm{g}$ food intake containing $2 \%$ flavangenol, $90 \mathrm{mg}$ flavangenol/day/mouse for 9 weeks) has been reported to suppress the atherosclerotic lesions without changing the levels of TBARS and other serum lipids except total cholesterol in ApoE-KO mice (10). In contrast, the present study has revealed that flavangenol administration $(6 \mathrm{ml}$ of $1 \mathrm{mg} / \mathrm{ml}$ flavangenol solution, $6 \mathrm{mg}$ flavangenol/day/mouse for 6 months) suppresses the atherosclerotic lesions accompanied with a significant increase of HDL-cholesterol and decrease of TBARS in B6.KOR-Apoe ${ }^{\text {shl }}$ mice. The dissimilar effects of flavangenol between the two studies may be explained by the differences in the doses (90 mg vs. $6 \mathrm{mg}$ ), durations (9 weeks vs. 6 months), delivery methods (food intake vs. drinking) and mouse strains (ApoE-KO vs. B6.KORApoe $^{\text {shl }}$ ) used.

Collectively, the present study has provided evidence that flavangenol, one of the most potent antioxidants, exerts an anti-atherosclerotic action on SHL and atherosclerotic B6.KOR-Apoeshl mice, possibly by increasing HDL-cholesterol levels and reducing lipid peroxide levels, thereby suppressing lipid accumulation (formation of atherosclerotic lesion) and inflammatory cell infiltration (chronic inflammation) in the intima of the aortic root. The effect of flavangenol on human atherosclerotic disorders and its mechanism should be further evaluated in the future.

\section{Acknowledgements}

This study was partially supported by a High Technology Research Center Grant from the Ministry of Education, Culture, Sports, Science and Technology of Japan, and the Research Institute for Diseases of Old Age, Juntendo University School of Medicine.

\section{References}

1. Breslow JL: Cardiovascular disease burden increases, NIH funding decreases. Nat Med 3: 600-601, 1997.

2. Li H, Witte K, August M, Brausch I, Armbrust U, Habermeier A, Closs EI, Oelze M, Munzel T and Forstermann U: Reversal of eNOS uncoupling and upregulation of eNOS expression lowers blood pressure in hypertensive rats. J Am Coll Cardiol 47: 2536-2544, 2006.

3. Hink U, Li H, Mollnau H, Olze M, Matheis E, Hartmann M, Skatchkov M, Thaiss F, Stahl RA, Warnholtz A, Meinertz T, Griendling K, Harrison DG, Forstermann U and Munzel T: Mechanisms underlying endothelial dysfunction in diabetes mellitus. Circ Res 88: e14-e22, 2001.

4. Burke A and Fitzgerald GA: Oxidative stress and smokinginduced vascular injury. Prog Cardiovasc Dis 46: 79-90, 2003.

5. Whitman SC: A practical approach to using mice in atherosclerosis research. Clin Biochem Rev 25: 81-93, 2004. 
6. Packer L, Rimbach G and Virgili F: Antioxidant activity and biologic properties of a procyanidin-rich extract from pine (Pinus Maritima) bark, pycnogenol. Free Radic Biol Med 27: 704-724, 1999

7. Nelson AB, Lau BH, Ide $\mathrm{N}$ and Rong Y: Pycnogenol inhibits macrophage oxidative burst, lipoprotein oxidation, and hydroxyl radical-induced DNA damage. Drug Dev Ind Pharm 24: 139-144, 1998.

8. Golanski J, Muchova J, Golanki R, Durackova Z, Markuszewski L and Watara C: Does pycnogenol intensify the efficacy of acetylsalicylic acid in the inhibition of platelet function? In vitro experience. Postepy Hig Med Dosw 60: 316-321, 2006.

9. Fitzpatrrick DF, Bing B and Rohdewald P: Endotheliumdependent vascular effects of Pycnogenol. J Cardiovasc Pharmacol 32: 509-515, 1998.

10. Sato M, Yamada Y, Matsuoka H, Nakashima S, Kamiya T, Ikeguchi $\mathrm{M}$ and Imaizumi K: Dietary pine bark extract reduces atherosclerotic lesion development in male ApoE-deficient mice by lowering the serum cholesterol level. Biosci Biotechnol Biochem 73: 1314-1314, 2009.

11. Auclair A, Milenkovic D, Besson C, Chuvet C, Gueux E, Morand C, Mazur A and Scalbert A: Catechin reduced atherosclerotic lesion development in apo E-deficient mice: a transcriptomic study. Atherosclerosis 204: e21-e27, 2009.

12. Miyazaki K, Makino K, Iwata E, Deguchi Y and Ishikawa F: Anthocyanins from purple sweet potato lpomoea batatas cultivar Ayamurasaki suppress the development of atherosclerotic lesions and both enhancements of oxidative stress and soluble vascular cell adhesion molecule-1 in apolipoprotein Edeficient mice. J Agric Food Chem 56: 11485-11492, 2008.

13. Catapano AL: Antioxidant effect of flavonoids. Angiology 48: 39-44, 1997.

14. Hertog MGL, Feskens EJM and Kromhout D: Antioxidant flavonols and coronary heart disease risk. Lancet 349: 699, 1997.
15. Putter M, Grotemeyer KH, Wurthwein G, Aragi-Niknam M, Watson RR, Hosseini S and Rohdewald P: Inhibition of smoking-induced platelet aggregation by aspirin and pycnogenol. Thromb Res 95: 155-161, 1999.

16. Matushima Y, Sakurai T, Ohnuki T, Tada N, Asoh Y and Tachibana M: Four strains of spontaneously hyperlipidemic (SHL) mice: phenotypic distinctions determined by genetic backgrounds. J Atheroscler Thromb 8: 71-79, 2001.

17. Matushima Y, Hayashi S and Tachibana M: Spontaneously hyperlipidemic (SHL) mice: Japanese wild mice with apolipoprotein E deficiency. Mamm Genome 10: 352-357, 1999.

18. Fearon IM and Faux SP: Oxidative stress and cardiovascular disease: novel tools give (free) radical insight. J Mol Cell Cardiol 47: 372-381, 2009.

19. Rader DJ and Daugherty A: Translating molecular discoveries into new therapies for atherosclerosis. Nature 415: 904-913, 2008.

20. Cathcart MK: Regulation of superoxide anion production by NADPH oxidase in monocytes/macrophages: contributions to atherosclerosis. Arterioscler Thromb Vasc Biol 24: 23-28, 2004.

21. Ferdinand KC: The importance of aggressive lipid management in patients at risk: evidence from recent clinical trials. Clin Cardiol 27: 12-15, 2004

22. Bittner V: Perspectives on dyslipidemia and coronary heart disease in women. J Cardiol 46: 1628-1638, 2005.

23. Gordon DJ, Probstfield JL, Garrison RJ, Neaton JD, Castelli WP, Knoke JD, Jacobs DR Jr, Bangdiwala S and Tyroler HA: Highdensity lipoprotein cholesterol and cardiovascular disease. Four prospective American studies. Circulation 79: 8-15, 1989.

24. Jan M, Meng S, Chen NC, Mai J, Wang H and Yang XF: Inflammatory and autoimmune reactions in atherosclerosis and vaccine design informatics. J Biomed Biotechnol 2010: 459798, 2010. 